\title{
GiG im Gespräch 2021/1
}

Sehr geehrte Kolleginnen und Kollegen,

liebe Mitglieder der Gesellschaft für interkulturelle Germanistik,

sehr geehrte Leserinnen und Leser der Zeitschrift für interkulturelle Germanistik,

als Allererstes möchte ich dieses Mal unserem hochgeschätzten Kollegen, langjährigem GiG-Mitglied, sehr verdienstvollem GiG-Vorstand und ausgezeichnetem Gesprächspartner Herbert Uerlings an dieser Stelle nochmals zu seinem besonderen Geburtstag ganz herzlich gratulieren, den er im vergangenen Jahr gefeiert hat. Im Namen der gesamten Gesellschaft für interkulturelle Germanistik übermittle ich Dir, lieber Herbert, unsere allerbesten Wünsche.

Ebenfalls sehr herzlich gratuliere ich unserer GiG-Preisträgerin, der renommierten amerikanischen Germanistin und Literaturwissenschaftlerin Leslie Allan Adelson, und unserem GiG-Preisträger Stefan Hermes. Beide teilen sich dieses Mal den Preis für erfahrene Forscherinnen und Forscher. Außerdem gratuliere ich ebenso herzlich Václav Smyčka, der den Preis für jüngere Forscherinnen und Forscher erhalten hat. Ausdrücklich danke ich an dieser Stelle allen Mitgliedern des Internationalen Ausschusses und des Wissenschaftlichen Beirats der GiG, die die Nominierungen begutachtet haben. Auf dieser Grundlage hat der Vorstand in seiner Videokonferenz am 10. Dezember 2020 intensiv und lange diskutiert und sich einhellig darauf verständigt, dass der GiG-Preis für etablierte Forscherinnen und Forscher dieses Mal aufgeteilt und jeweils zur Hälfte an Leslie Allan Adelson und Stefan Hermes verliehen wird und der für jüngere Forschende an Václav Smyčka aus Tschechien. Wie freuen uns, dass wir mit den beiden GiG-Preisen wieder eine ausgezeichnete Fachvertreterin und ausgezeichnete Fachvertreter ehren konnten. Den Termin für die offizielle Preisverleihung konnten wir unter den derzeit nach wie vor gegebenen pandemischen Unwägbarkeiten noch nicht endgültig festlegen, aber wenn möglich, sollte sie in diesem Jahr im Rahmen der IVG-Tagung stattfinden. Natürlich werden Sie dann umgehend die Details erfahren.

In der Rubrik GiG im Gespräch des vorliegenden ZiG-Heftes greife ich nun ein Thema auf, das aus meiner Sicht unsere Aufmerksamkeit verdient. Denn vor wenigen Wochen hat das Auswärtige Amt der Bundesrepublik Deutschland auf seiner Website eine Unterlage veröffentlicht, in der eine neue Strategie in der Außen- und Kulturpolitik skizziert wird (vgl. Auswärtiges Amt 2020). Im Zentrum steht nunmehr der Begriff einer >Science Diplomacy< respektive >Wissenschaftsdiplomatieく. Viel von dem, was auch die GiG mit ausmacht, nämlich u.a. »akademische Mobilität und wissenschaft- 
liche Kooperationen« werden seitens des Auswärtigen Amtes in ihrer strategischen Bedeutung für eine »nachhaltige deutsche Außenpolitik« akzentuiert (ebd.). In der Wissenschaftsdiplomatie Deutschlands soll die Vernetzung aktiv mitgestaltet und ein weltweiter Einsatz für Freiheit von Wissenschaft und Forschung geleistet werden.

Nun ist es gewiss so, dass nicht nur aus wissenschaftlicher Sicht die vielen von uns in praktisch allen Belangen zum Grundsatz gewordene Haltung der Skepsis auch hier angebracht sein dürfte. Desto mehr, so meine ich, sollten wir uns über solche Entwicklungen gut informiert halten, nicht zuletzt, weil Positionierungen des Auswärtigen Amtes u.a. auch für das Goethe-Institut, den DAAD und die Alexander von Humboldt Stiftung Maßstab setzend sind. Sehen wir uns die Dinge also etwas genauer an und unterziehen das auf der Website des AA abrufbare Strategiepapier "Science Diplomacy« vom Dezember 2020 gewissermaßen einer gemeinsamen Lektüre.

Die besagte neue Strategie wird einleitend mit der Absicht verbunden, »Wissenschaft als notwendige Bedingung demokratischen Handelns zu fördern« (ebd.), welches seinerseits auf einem Diskurs informierter Bürgerinnen und Bürger und ihrer Vertreterinnen und Vertreter beruhe und als Modell wissenschafts- und evidenzbasierter Politik in der Tradition der Aufklärung stehe. Diese Tradition wird mit implizitem Kant-Zitat als Ausgang aus der Unmündigkeit bezeichnet und soll dem Strategiepapier zufolge nun neu belebt werden. Denn Freiheitsräume von Wissenschaft und Gesellschaft seien zunehmend bedroht, würden zunehmend kommunikativ unterlaufen und faktisch u.a. durch eine mangelnde Ausstattung mit Ressourcen bedroht. Entsprechenden autoritären Narrativen sei zu begegnen mit der »Stärke Europas, wissensbasiert verantwortliche politische Entscheidungen zu treffen und Wissenschaft ebenso wie Kunst und Kultur - als Freiheits- und Erkenntnisraum für gesellschaftliche Entscheidungen zu pflegen« (ebd.), die identitätsstiftend sowohl nach innen als auch europaweit und global nach außen wirken solle.

Neben diesem politischen Auftrag, demokratische Strukturen zu stärken, werden verschiedene Themenbereiche aufgezählt, die im Zentrum wissenschaftlichen Arbeitens stehen sollen, darunter solche, die mit sozial- und geisteswissenschaftlichen $\mathrm{Zu}$ ständigkeiten verbunden sind, wie die Auswirkungen menschlichen Handelns auf die natürliche Lebenswelt, Fragen der Diversität in den nationalen Gesellschaften, Wissenschaftskommunikation »mit einer engagierten Bürgergesellschaft« (ebd.), Fluchtursachen und Migration. Ausdrücklich hervorgehoben wird die Friedenspolitik unter besonderer Berücksichtigung von Wissenskommunikation und Wissenstransfer; dabei sei den Empfehlungen des Wissenschaftsrates zur Friedens- und Konfliktforschung zu folgen (vgl. Wissenschaftsrat 2019).

Auf diese Analyse des Wissenschaftsrates möchte ich Ihrer Aufmerksamkeit lenken, wenn Sie sich für Themen im Zusammenhang von Frieden und Konflikt interessieren. In diesen informativen Empfehlungen wird ein aktueller Bericht zur disziplinären Ausbildung, Lehr- und Forschungslage sowie internationalen Kooperationspartnern in diesem Gebiet vorgelegt und u.a. auf seine Interdisziplinarität aufmerksam gemacht, wobei auch die Geschichts-, Geistes-, Kommunikations-, Sprachund Literaturwissenschaften sowie natürlich auch interkulturelle Kompetenzen und Migrationsforschung Erwähnung finden (vgl. ebd.: 14, 43, 46-48, 54f., 71, 114). Aus meiner Sicht wird zu Recht mit Bedauern bemerkt, dass die Friedens- und Konfliktforschung u.a. in den Kommunikations-, Medien- und Kulturwissenschaften gar nicht institutionell verankert sei, so dass z.B. Radikalisierungsprozesse nur unzureichend 
erfasst werden könnten (vgl. ebd.: 47). Ausdrücklich wird jedoch auch ein Punkt angesprochen, der Sie vielleicht bei der Lektüre meiner bisherigen Skizze schon ein wenig unruhig gemacht haben könnte, nämlich die Frage der Unabhängigkeit der Wissenschaft angesichts einer möglichen mangelnden Distanz seitens der Wissenschaft ebenso wie einer drohenden Vereinnahmung seitens politischer Instanzen und Zielsetzungen (vgl. ebd.: 19-21).

Für eine vertiefte Auseinandersetzung mit dem Konzept Science Diplomacy ist der Blick auf Formen der Institutionalisierung ebenso wie historische Hintergründe zu richten. Zur Institutionalisierung gehören die Gründung des Center for Science Diplomacy unter dem Dach der American Association for the Advancement of Science (vgl. AAAS o.J.) und in diesem Rahmen die Einrichtung des Onlineperiodikums Science \& Diplomacy (vgl. https://www.sciencediplomacy.org/). Wenn man so will, erfährt die Konzeption Nobilitierung seitens einer der weltweit ältesten wissenschaftlichen Akademien, nämlich der traditionsreichen Royal Society, die 1660 gegründet wurde, Wissenschaft von Anfang an international begriff und 1723 die Position eines Foreign Secretary einrichtete (vgl. Poliakoff 2015). 2009 fand ein gemeinsam mit der AAAS organisiertes zweitägiges Treffen statt, das den Titel New frontiers in science diplomacy trug. Es ist unter demselben Titel dokumentiert und im Netz abrufbar (vgl. The Royal Society 2010). Einleitend wird betont:

Science diplomacy is not new, but it has never been more important. Many oft he defining challenges oft he 21st century - from climate change and food security, to poverty reductionn and nuclear disarmament - have scientific dimensions. No one country will be able to solve these problems on ist own. The tools, techniques and tactics of foreign policy need to adapt to a world of increasing scientific and technical compexity. (Ebd.: v)

Ähnlich wird das Konzept auch seitens des Deutschen Bundestages begründet, der dabei die Aspekte von Sprache und Kultur besonders betont: »Die frühzeitige Vermittlung der Chancen, die kulturelle Diversität und Kooperation zwischen den Kulturen bieten, um gemeinsame Probleme durch gemeinsames Handeln zu lösen, ist eine Aufgabe, die in besonderer Weise von den Deutschen Auslandsschulen und den PASCHSchulen wahrgenommen wird« (Deutscher Bundestag 2020: 4).

Nun, wie ich oben bereits andeutete, werden diese Entwicklungen mit kritischem Interesse und angemessener - mehr oder weniger wohlwollender - Skepsis zu betrachten sein. Im letzten Heft der ZiG schrieb ich, dass wir es womöglich mit einer Situation zu tun hätten, »in der es parallel sowohl zu Entwicklungen neuer Formen von gewissermaßen sautoritär verengten Kommunikationsräumen als auch zunehmend zur Horizonterweiterung im Sinn einer Vergrößerung unserer kommunikativen Responsivität« komme (Schiewer 2020: 249). Damit würde internationale Kommunikation jedoch - auch oder gerade bei Themen wie Klimaschutz, die als "gemeinsame Probleme durch gemeinsames Handeln zu lösen« seien - noch komplexer als immer schon. Dies hat sich in der Vergangenheit u.a. daran gezeigt, wie viele Jahre verstrichen, bis es am 12. Dezember 2015 auf der internationalen Klimakonferenz in Paris zum Beschluss eines Abkommens kam. Ich meine, dass wir unsere Expertise in der Interkulturellen Germanistik und in Interkulturellen Austauschprozessen hier einbringen können und auch sollten. 
Schließlich möchte ich an dieser Stelle auf die seit 2015 bestehende Philipp Schwartz-Initiative für gefährdete Wissenschaftler*innen aufmerksam machen, die als Sonderprogramm für die Aufnahme und Integration gefährdeter Wissenschaftlerinnen und Wissenschaftler in Deutschland einen Teilbereich der Wissenschaftsdiplomatie darstellt (vgl. Alexander von Humboldt-Stiftung o.J.).

Wie immer grüßt Sie sehr herzlich

Ihre Gesine Lenore Schiewer

\section{Literatur}

AAAS (Hg.; o.J.): Center for Science Diplomaca, Homepage; online unter: https://www. aaas.org/programs/center-science-diplomacy [Stand: 1.2.2021].

Alexander von Humboldt-Stiftung (Hg.; o.J.): Philipp Schwartz-Initiative, Homepage; online unter: https://www.humboldt-foundation.de/bewerben/foerderprogramme/philipp-schwartz-initiative [Stand: 1.2.2021].

Auswärtiges Amt (Hg.;2020): Science Diplomacy, Dezember 2020; online unter: https:// www.auswaertiges-amt.de/blob/2423206/a2086c45807120c7b5842ba5055649eb/ 201203-science-diplomacy-strategiepapier-data.pdf [Stand: 1.2.2021].

Deutscher Bundestag (Hg.; 2020): Antrag der Fraktionen der CDU/CSU und SPD. Die Auswärtige Kultur- und Bildungspolitik im Wandel - Neue Bedingungen und Herausforderungen für zeitgemäßes Handeln, Drucksache 19/16834; online unter: https://dip21.bundestag.de/dip21/btd/19/168/1916834.pdf [Stand:1.2.2021].

Poliakoff, Martyn (2015): The Royal Society, the Foreign Secretary, and International Relations. In: Science \& Diplomacy 4, H. 1; online unter: https://www.sciencediplomacy.org/letter-field/2015/royal-society-foreign-secretary-and-internationalrelations [Stand: 1.2.2021].

Schiewer, Gesine Lenore (2020): GiG im Gespräch 2020/2. In: Zeitschrift für interkulturelle Germanistik 11, H. 2, S. 247-249.

The Royal Society (Hg.; 2010): New frontiers in science diploimacy. Navigating the changing balance of power. RS Policy document 1/10; online unter: https://royalsociety.org/ /media/royal_society_content/policy/publications/2010/4294969468. pdf [Stand: 1.2.2021].

Wissenschaftsrat (Hg.; 2019): Empfehlungen zur Weiterentwicklung der Friedensund Konfliktforschung, 12. Juli 2019; online unter: https://www.wissenschaftsrat. de/download/2019/7827-19.pdf?__blob=publicationFile\&v=2 [Stand: 1.2.2021]. 